\title{
Aspetti Pragmatici dell'Enunciato in un ATto di Comunicazione in Ambiente INTERCULTURALE
}

\author{
Eva Kú́moví*
}

ABSTRACT: Nel contributo vengono considerati alcuni aspetti pragmatici dell'enunciato nell'atto di comunicazione. Tra i fattori della situazione comunicativa sono presi in considerazione i partner della comunicazione e il rapporto tra di loro, il contesto extralinguistico e lo scopo della comunicazione. I fattori della situazione comunicativa, messi in relazione con i principi della comunicazione di G. Leech, determinano sia l'utilizzo di strutture linguistiche in una concreta situazione comunicativa sia lo svolgimento dell'atto comunicativo non solo nell'ambito di una sola comunità linguistica ma anche tra due lingue diverse.

PAROLE CHIAVE: principi pragmatici; situazione comunicativa; lingua italiana.

RESUMO: No artigo são considerados alguns aspectos pragmáticos do enunciado no ato de comunicação. Entre os fatores da situação comunicativa são levados em conta os parceiros da comunicação e sua relação, o contexto extralinguístico e a finalidade da comunicação. Os fatores da situação comunicativa, relacionados com os princípios da comunicação de G. Leech, determinam tanto a utilização de estruturas linguísticas em uma concreta situação comunicativa, quanto o

*Docente Università della Slesia di Opava (Repubblica Ceca). 
desenvolvimento do ato comunicativo, não apenas no âmbito de uma única comunidade linguística, mas também entre duas línguas diferentes.

PALAVRAS-CHAVE: principi pragmatici; situazione comunicativa; língua italiana.

ABSTRACT: The article deals with some pragmatic aspects of the utterance in the act of communication, i.e. the factors of the communicative situation and pragmatic principles. For the factors of the communicative situation the relation between the speaker and the addressee, the context of the utterance and the goal of the utterance are given. These factors are considered with respect to the Leech's conversational principles of the interpersonal and the textual rhetoric that determine the usage of particular linguistic structures in the act of communication not only within one and the same linguistic community but also when speakers of two different languages get into contact.

KEY WORDS: pragmatic principles; communicative situation; Italian language. 


\section{Introduzione}

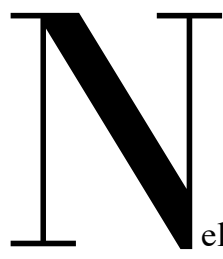

el contributo vengono considerati alcuni aspetti pragmatici dell'enunciato in un certo atto di comunicazione, pensando in particolare ai fattori della situazione comunicativa e ai principi della conversazione. I fattori della situazione comunicativa, messi in relazione con i principi della conversazione, condizionano sia l'utilizzo di un concreto strumento linguistico in una situazione comunicativa concreta, sia lo svolgimento dell'atto comunicativo, e ciò non solo nell'ambito di una sola comunità linguistica, ma molto fortemente anche tra parlanti di madrelingua diversa. Le considerazioni su questi aspetti pragmatici dell'enunciato sono qui condotte con l'obiettivo di offrire una presentazione delle questioni e nozioni che si incontrano nell'analisi della struttura della frase, da studiare a tre livelli: grammaticale, semantico e pragmatico; nonché con l'ulteriore scopo di dimostrare come sia facile il passaggio da un livello 
all'altro. Vogliamo cioè porci la questione non solo di come una frase si costruisca, ma anche di quali siano i significati attribuibili a un enunciato in una concreta situazione comunicativa, in quanto "... only through the study of language in use are all the functions of language, ..., brought into focus” (HALLIDAY, 1970, p. 145).

Nelle nostre considerazioni, a questo proposito, ci avvaliamo prima di tutto di una delle fonti che consideriamo più affidabili, cioè i Principles of Pragmatics di G. Leech (1983). Secondo lo studioso, l'uso di una forma o struttura linguistica in una concreta situazione comunicativa viene condizionato dai principi della retorica interpersonale e di quella testuale. Egli riassume la relazione tra i due livelli dell'analisi linguistica, cioè il livello grammaticale e quello pragmatico, in otto postulati, ed è opportuno citare qui il terzo: "The rules of grammar are fundamentally conventional. The principles of general pragmatics are fundamentally non-conventional, i.e. motivated in terms of conversational goals" (LEECH, 1983, p. 5). La conversazione, nella quale vigono i principi della retorica interpersonale può ricordare un gioco diretto da regole puramente convenzionali, così come le considera Wittgenstein nel suo concetto di "gioco linguistico", paragonando l'attività linguistica in una certa situazione con il gioco degli scacchi: un pezzo sulla scacchiera ha un valore solo in rapporto a tutti gli altri pezzi, così come una parola in un enunciato acquista un significato solo in rapporto con gli altri elementi di esso in quella concreta situazione comunicativa (cfr. WITTGENSTEIN, 1993, p. 40).

Dal livello grammaticale a quello pragmatico, si può arrivare solo attraverso il livello semantico, anche se non è facile fare una netta distinzione tra il significato semantico e il significato pragmatico. Da questo punto di vista è utile avvalersi di due concetti di Leech: il concetto di sense per il significato semantico e il concetto di pragmatic force per il significato pragmatico, legato a una concreta situazione comunicativa, sempre dipendente dall'intenzione comunicativa del parlante. Se qualcuno dice Qui si fuma troppo, la sua intenzione comunicativa non è solo quella di far capire all'interlocutore il senso di quello che dice ma anche di indurlo $\mathrm{ad}$ un'azione. Esortarlo cioè ad aprire la finestra o a smettere di fumare, raggiungendo un effetto concreto.

In seguito parleremo più dettagliatamente di alcuni aspetti pragmatici dell'enunciato in un atto di comunicazione. Prima vengono considerati i fattori della situazione comunicativa, quali i partecipanti alla conversazione, l'intenzione comunicativa del parlante e il contesto (parte 2); dopo si passa al funzionamento di alcuni principi della conversazione (parte 3). Essi svolgono un ruolo decisivo non solo nell'ambito di una concreta comunità linguistica analizzata isoltamente, 
ma soprattutto lì dove si incontrano persone appartenenti per madrelingua a comunità linguistiche e culture diverse, il che condiziona l'uso delle forme e strutture linguistiche proprie della lingua della conversazione esaminata. Si cercherà, per finire, di mettere in relazione i fattori della situazione comunicativa con i principi della comunicazione. Essi, nell'interazione, determinano il modo di "fare cose con le parole".

\section{Fattori della situazione comunicativa}

I fattori della situazione comunicativa presi in considerazione per le nostre osservazioni sono: i partecipanti alla conversazione, le questioni legate all'espressione dell'intenzione comunicativa del parlante, e il contesto (non solo linguistico). In un atto di comunicazione, questi fattori operano interagendo tra loro.

Per partecipanti alla comunicazione si intendono il parlante e la persona alla quale egli si rivolge, cioè l'interlocutore, e considerati sulla base del rapporto che intercorre tra di loro, del loro ruolo sociale, dell'età, del grado di istruzione o della professione. In ogni caso, è importante che tutti e due percepiscano e interpretino la situazione comunicativa nello stesso modo.

Per esprimere con chiarezza la propria intenzione comunicativa e per raggiungere lo scopo della conversazione, il parlante deve prendere in considerazione i fattori della situazione comunicativa menzionati sopra. A seconda del rapporto che intercorre tra gli interlocutori, il contesto l'enunciato può avere la funzione di alcuni atti illocutivi. Pronunciando un enunciato assertivo come:

\section{Verrò alle tre}

l'intenzione comunicativa del parlante può essere quella di informare l'interlocutore su un'azione futura prevista, per esempio nella situazione in cui il direttore informi la segretaria del proprio arrivo in ufficio. Questo enunciato può invece essere facilmente percepito come una promessa nella situazione in cui il figlio debba rassicurare la madre sul proprio rientro a casa. Esso può addirittura fungere da minaccia tra due nemici, ecc. Se invece il parlante pronuncia un enunciato interrogativo come:

2. Perché non sei venuto? 
la sua intenzione comunicativa è quella di indurre l'interlocutore a rispondere, con lo scopo di ottenere dall'interlocutore un'informazione. In questo caso concreto il parlante vuole sapere la ragione per cui l'interlocutore non sia venuto. Una frase interrogativa del genere è quindi classificabile come una domanda pura. A questo proposito è opportuno distinguere gli enunciati interrogativi che svolgono la funzione di domanda da quelli che possono avere la funzione di enunciati “pseudo-interrogativi”. Questo stesso enunciato, per esempio, potrebbe essere pronunciato dal parlante con l'intenzione di esprimere il suo disaccordo o la sua meraviglia.

L'intenzione comunicativa del parlante può essere ancora quella di indurre l'interlocutore a un'azione non verbale, di indurlo cioè 'a compiere oppure a non compiere un'azione non verbale o verbale esplicitamente menzionata nell'enunciato" (STATI, 1982, pp. 68 e 75). Pronunciando un enunciato direttivo come:

\section{Fai come faccio io}

il parlante vuole ordinare qualche cosa all'interlocutore. L'enunciato è quindi un ordine, ma può essere percepito anche come un consiglio. A proposito degli enunciati direttivi bisogna ricordare gli "pseudoimperativi", la cui funzione non è quella di indurre l'interlocutore a un'azione (cfr. ASCOLI, 1978). Nell'enunciato:

\section{Calmatevi, calmatevi}

il parlante vuole tranquillizzare l'interlocutore. In questo caso si ha il passaggio dall'imperativo direttivo a quello non direttivo, che si osserva allorquando con esso non si esprime una richiesta, e quindi è considerato uno "pseudoimperativo".

Come dimostrano gli esempi precedenti, per ogni tipo di frase si presuppone uno schema modale che tuttavia può cambiare facilmente, comportando il cambiamento della funzione comunicativa dell'enunciato. A questo proposito possiamo paragonare una serie di enunciati che, attraverso la trasposizione delle categorie grammaticali del verbo, cioè di modo o di persona $\mathrm{e}$ di tempo, e assieme all'uso di una particella modale, esprimono non solo un diverso grado di indignazione del parlante, ma determinano il cambiamento dell'atto illocutivo:

1 Su alcuni tipi di imperativo non direttivo cfr. Klímová, 2004. 
${ }^{\circledR}$ Non rinfacciarmelo.

${ }^{\circledR}$ Me lo stai rinfacciando di nuovo.

® Vuoi rinfacciarmelo?

${ }^{\circledR}$ Rinfacciarmelo!

${ }^{\circledR}$ Non rinfacciarmelo o ti faccio vedere. proibizione

rimprovero

indignazione

meraviglia

minaccia

La polifunzionalità di un enunciato è determinata dall'intreccio dei fattori della situazione comunicativa citati sopra con i principi della conversazione di cui parleremo nella sezione successiva.

Delineare il contesto in cui si svolge la conversazione è piuttosto complicato perché, oltre al contesto linguistico, cioè quello che è stato detto prima a proposito del tema della conversazione, e al contesto situazionale determinato dal momento, nonché dal luogo in cui la conversazione si svolge, entra in gioco tutto quello che chi partecipa alla conversazione "sa" a proposito del tema ${ }^{2}$. Siamo del parere che questo tipo di contesto sia determinato anche dalla capacità di una concreta persona di tenere in considerazione gli elementi della realtà extralinguistica, soprattutto in un ambiente che non sia quello della sua madrelingua. Bisogna aggiungere che il contesto, sia quello linguistico sia quello situazionale, assieme all'ordine dei costituenti, la struttura semantica e l'intonazione, è uno dei fattori della struttura informativa dell'enunciato stabiliti da J. Firbas ${ }^{3}$. Quindi ci torneremo più avanti trattando il rapporto tra il contesto e uno dei principi della conversazione, il Processability Principle (principio della processabilità).

\section{I principi della conversazione e il concetto pragmatico di retorica}

Prima di presentare i principi della conversazione, bisogna citare il concetto di retorica, uno dei concetti più importanti nelle considerazioni sulla funzione della lingua, con la quale Leech intende "the effective use of language in its most general sense, applying it primarily to everyday conversation ..." (1983, p. 15). Il parlante usa una lingua esprimendo la propria intenzione comunicativa con lo scopo di produrre "a particular effect in the mind of the hearer" (p. 15). Il concetto di retorica comprende due componenti: la retorica interpersonale e quella testuale, tutte e due comprendenti alcuni principi della conversazione.

Nell'ambito della retorica interpersonale cooperano due principi: (a) il principio di cooperazione, ripartito a sua volta in alcune massime: massima della qualità (sii sincero),

2 Per la determinazione del contesto cfr. Firbas, 1979, p. 31.

3 Sui fattori della struttura informativa dell'enunciato cfr. Klímová 2007. 
massima della quantità (fornisci l'informazione necessaria, né più, né meno), massima della relazione (sii pertinente all'argomento, al tema della conversazione), massima del modo (sii chiaro, non essere ambiguo $)^{4}$; e il principio di cortesia, che include massima del tatto, massima della generosità, massima dell'approvazione, massima delal modestia, massima dell'assecondamento, massima della simpateticità (cfr. LEECH, 1983, p. 132). Le massime del principio di cortesia, come quelle del principio di cooperazione, si intrecciano operando in interazione. I partecipanti alla conversazione, per garantirne un andamento 'normale', devono accettarle e rispettarle, adattandosi alla situazione comunicativa. Se, per esempio, il parlante dice Mi passi il sale? un interlocutore "normale" percepisce l'enunciato non come domanda alla quale rispondere sì o no, ma come una richiesta alla quale reagire passando al parlante il sale, anche se, negli scambi conversazionali, le infrazioni di queste regole conversazionali sono molto frequenti.

Da una parte, per raggiungere lo scopo della conversazione, bisogna rispettare sia le massime del principio di cooperazione sia quelle del principio di cortesia. Dall'altra parte può succedere che esse contrastino: se un partecipante dice Passami il sale, quello che dice è preciso, rilevante, e lo scopo della comunicazione è chiaro. Invece, la forma, cioè come il parlante formula la richiesta, non è sufficientemente cortese da essere impiegata rivolgendosi a qualsiasi interlocutore. Si ha, cioè, una distinzione netta tra gli obiettivi illocutivi e il traguardo sociale (cfr. LEECH, 1983, p. 17).

Per le nostre considerazioni il principio di cooperazione è di importanza marginale, perché l'applicazione delle sue massime è legata piuttosto alla questione di "che cosa" il parlante voglia dire, mentre il principio di cortesia, di cui vogliamo occuparci più dettagliatamente, è legato alla questione di "come" parlare; è legato, cioè, all'uso delle strutture di una lingua concreta. Tuttavia, il principio di cortesia non si manifesta solo al livello della lingua. Esso è ancorato alle norme e alle usanze tradizionali di comportamento che possono essere, da una cultura all'altra, molto diverse. Così, per esempio, nel caso dei complimenti, uno statunitense rispetterà poco la massima della modestia perché per la cultura statunitense è importante incoraggiare le persone a fare; i giapponesi, invece, tengono molto a questa massima minimizzando ogni lode ${ }^{5}$. Diverse culture percepiscono diversamente anche la massima del tatto (Tact Maxim). Nell'ambiente ceco, per esempio, è normale fare domande personali, il che viene considerato sgarbato nell'ambiente anglosassone. I cechi, cioè, violano la massima del tatto. Si considerano invece beneducati rifiutando quello che con sincerità gli viene offerto, nel corso di una visita,

4 A questo proposito, si vedano: Grice, 1975, pp. 204-206; Leech, 1983, p. 8 e p. 79; Bettoni, 2006, p. 80.

5 Sulla massima della modestia, si veda anche Bettoni, 2006. 
aspettando, paradossalmente, la seconda offerta. Nell' ambiente anglosassone, se si rifiuta qualche cosa l'offerta non viene ripetuta. Al contrario, nell'ambiente italiano rifiutare non sempre è considerato segno di buona educazione.

La retorica testuale, così come la retorica interpersonale, include alcuni principi: Clarity Principle (principio di chiarezza), Economy Principle (principio di economia) e Processability Principle (principio di processabilità) (cfr. LEECH, 1983, p. 16), nell'ambito del qual è di grande importanza per le nostre considerazioni la "end-weight maxim": applicandola nel discorso il parlante parte dall'informazione nota collocando quella nuova verso la fine della frase. Si tratta cioè dell'applicazione di quell'ordinamento lineare in cui opera il principio della struttura informativa dell'enunciato. In alcune lingue, per esempio in inglese, l'ordine delle parole, invece, è determinato dal principio grammaticale: la posizione nella frase è legata alla sua funzione sintattica e l'ordine delle parole soggetto-verbo-complemento oggetto è quindi fisso. In altre lingue, per esempio il ceco e in misura considerabile anche in italiano, l'ordine delle parole è maggiormente determinato dal principio della struttura informativa: in queste lingue si osserva una forte tendenza a collocare l'elemento rematico verso la fine dell'enunciato. E' l'esempio successivo ad illustrare questa differenza:

5. Ce l'ha appena detto il signor Black.

Mr Black here has just told us.

Nelle lingue in cui la "end-weight maxim” non è operante, è prima di tutto l'uso dell'articolo a svolgere una funzione importante nella struttura informativa dell'enunciato. Ne parleremo più avanti.

\section{I fattori della situazione comunicativa in relazione con i principi della conversazione}

In questa sezione si presentano alcune strutture linguistiche prendendo in considerazione $\mathrm{i}$ fattori della situazione comunicativa messi in relazione con i principi della conversazione dal punto di vista delle differenze interculturali che intercorrono tra due comunità linguistiche. In seguito vogliamo dedicare attenzione ad alcuni elementi linguistici che nel passaggio da una lingua all'altra sono, a proposito degli aspetti pragmatici menzionati sopra, i più degni di nota .

La maniera in cui i partecipanti al discorso rispettano il principio di cortesia si riflette nel modo in cui il parlante si rivolge all'altra persona, cioè la distinzione Tu/Lei. In ceco questa distinzione viene espressa dalla forma del verbo, la $2^{\mathrm{a}}$ singolare opposta alla $2^{\mathrm{a}}$ plurale, e dai 
pronomi allocutivi ty/vy, proprio come $t u / v o u s$ in francese. Rispettare la distinzione Tu/Lei in italiano, per uno studente ceco, non è quindi difficile; può invece esserlo per uno studente inglese, dove questa distinzione non è affidata né alla forma del verbo né al pronome allocutivo. La mancanza di una forma linguistica per fare una distinzione netta tra il rivolgersi formale o informale all'interlocutore può sembrare svantaggiosa. Invece può essere anche molto conveniente: non dovendo e non potendo distinguere formalmente tu e Lei/voi, gli inglesi ai cechi e agli italiani sembrano informali nel loro comportamento e, allo stesso tempo, sufficientemente formali per mantenere un certo distacco dall'interlocutore. Al contrario, nel contesto italiano sembra oggi sempre più forte la tendenza a dare del tu anche nelle situazioni in cui sarebbe stato ancora poco tempo fa "buona educazione" dare del lei. Se una commessa, in Italia, dà del tu ad una cliente straniera sconosciuta, è sgarbata o è semplicemente un segnale di confidenza o amichevolezza, o di mancanza di stima verso gli stranieri? Da parte sua può anche essere una strategia commerciale: un comportamento informale favorisce la vendita.

Sono degne di nota strutture di cortesia con verbi modali come strumento lessicale per indicare un significato modale, cioè un atteggiamento del parlante: esse, riguardo all'applicazione del principio di cortesia, possono svolgere un ruolo importante perché sono uno strumento efficace per esprimere differenze nell'atteggiamento del parlante. Non è un caso che in inglese siano numerose: la necessità di esprimere un certo grado di cortesia o formalità è legata direttamente al loro uso. In altre parole, lì dove in italiano e in ceco per distinguere una situazione formale da una non formale vengono adoperati strumenti flessivi, cioè la forma del verbo e i pronomi allocutivi, in inglese sono proprio le strutture con un verbo modale a svolgere questa funzione (per es. Could you ...?, Will you ...? Would you mind ...? ecc).

A proposito delle strutture con i verbi modali è utile ricordare l'opposizione perfetto imperfetto del verbo dovere in italiano, opposizione che non esiste né in ceco né in inglese. Se il parlante usa l'imperfetto nella frase Non dovevi farlo esprime il disaccordo e la funzione dell'enunciato è rimprovero. Se invece dice Non hai dovuto farlo indica che l'azione non si è svolta, perché non era necessario compierla. La funzione dell'enunciato è asserzione. In ceco e in inglese bisognerebbe, per esprimere questi significati modali, usare verbi modali diversi: il passato del verbo modale mít (avere) in ceco e il verbo modale should in inglese per il disaccordo (Non dovevi farlo. / Neměl jsi to dělat. / You should not have done it.); il passato del verbo modale muset (dovere) in ceco e had to in inglese per esprimere il significato di necessità (Non hai dovuto farlo. / Nemusel jsi to dělat. / You did not have to do it.) 
Confrontiamo inoltre le frasi seguenti per evidenziare la funzione del modo verbale, uno strumento grammaticale nell'indicazione che indica l'intenzione comunicativa del parlante:
a. Accompagnami a casa.
b. Mi accompagni a casa, vero?
c. Mi accompagneresti a casa?
d. Potresti accompagnarmi a casa?

imperativo

indicativo

condizionale

condizionale del verbo modale

La frase $a$ ha il carattere imperativo e l'interlocutore si sentirà/potrebbe sentirsi obbligato a fare quello che gli è chiesto. La frase $b$ è un enunciato dichiarativo che non costringe esplicitamente l'interlocutore a fare quello che gli è chiesto. È quindi più cortese rispetto all'enunciato direttivo $a$. Le frasi $c$ e $d$, essendo enunciati interrogativi, gli danno addirittura la possibilità di dire di no, gli danno cioè la possibilità di rifiutare. Usando un enunciato interrogativo, il parlante richiede qualche cosa indirettamente e anche più cortesemente. In altre parole, nel modo indiretto di esprimersi è evidente l'impiego del principio di cortesia.

L'ultimo elemento da commentare è il rapporto del contesto, linguistico e situazionale, con l'applicazione della "end-weight maxim" del Processability Principle. Nelle lingue ad ordinamento lineare delle quali opera il principio della struttura informativa, come il ceco, si osserva, grazie al caso morfologico, un ordine dei costituenti che è quasi del tutto libero, con una forte tendenza a spostare verso la fine della frase quell'elemento che funge da rema (cfr. KLÍMOVÁ, 2013, p. 49); in italiano, privo tra l'altro di caso morfologico, è normale, cioè sintatticamente non marcato, l'ordine $S$ - $V$ - $O$, le deviazioni da esso sono considerate sintatticamente marcate, ma sono possibili e abbastanza frequenti (cfr. KLÍMOVÁ, 2002). In inglese, l'ordine delle parole è controllato dal principio grammaticale. Nelle lingue di quel genere è l'articolo, determinativo e indeterminativo, a svolgere il ruolo determinante nell'atto di indicare un elemento come ricavabile oppure non ricavabile dal contesto. Nella frase inglese A boy came into the room il soggetto a boy occupa la posizione iniziale. Essendo accompagnato dall'articolo indeterminativo funge da rema dell'enunciato. Nella traduzione in ceco Do místnosti vstoupil chlapec, il soggetto rematico chlapec (ragazzo) occupa la posizione alla fine della frase. Invece nella traduzione ceca della frase inglese The boy came into the room con il soggetto the boy indicato dall'articolo determinativo come tema occuperebbe la posizione iniziale, cioè Chlapec vstoupil do místnosti. 


\section{Conclusioni}

Nelle nostre considerazioni sugli aspetti pragmatici dell'enunciato nel quadro dell'atto di comunicazione sono stati presentati i fattori della situazione comunicativa assieme all'applicazione di alcuni principi della conversazione. Si è cercato di evidenziare come essi siano importanti per un andamento naturale della comunicazione tra persone che appartengono ad una concreta comunità linguistica ma anche tra quelle appartenenti a comunità e culture diverse. Per raggiungere lo scopo nella comunicazione, i partecipanti alla situazione comunicativa, in un dato contesto, devono rispettare i principi della conversazione: il principio di cooperazione ed il principio di cortesia. In altre parole, sia il parlante sia l'interlocutore devono adattarsi alla situazione comunicativa scegliendo degli strumenti linguistici che corrispondano al rapporto che intercorre tra di loro e l'uso dei quali renda possibile la buona riuscita di una conversazione.

I concetti linguistici attinenti alla teoria dei principi pragmatici fanno parte della cornice teorica nella quale si inserisce lo studio della sintassi funzionale, così come ne fanno parte, assieme alla struttura grammaticale della frase, anche la teoria degli atti linguistici e quella della struttura informativa dell'enunciato.

Nelle nostre considerazioni si è cercato anche di dimostrare come sia importante per ottenere un andamento naturale della comunicazione rispettare i principi della conversazione nella scelta di forme e strutture linguistiche proprie alla lingua in questione.

\section{Riferimenti bibliografici}

ASCOLI, C. Some pseudo-imperatives and their communicative function in English. In Folia Linguistica, XII: 405-416, 1978.

BETTONI, C. Usare un'altra lingua. Guida alla pragmatica interculturale. Roma, Laterza, 2006.

FIRBAS, J. A functional view of 'ordo naturalis'. In Brno Studies in English 13, 1979, pp. 29-59.

. Functional sentence perspective in written and spoken communication. Cambridge:

Cambridge University Press, 1992.

GRICE, P. H., Logic and Conversation. In COLE, P.; MORGAN, J.L. (eds.). Syntax and semantics 3: Speech acts. New York, Academic Press, 1975, pp. 41-58 (trad. it. Logica e conversazione. In SBISÀ, M. (ed.), Gli atti linguistici, Milano: Feltrinelli, 1978, pp. 199-219).

HALLIDAY, M. A. K. Language Structure and Language Function. In LYONS, J. New Horizons in 
linguistics, London, 1970, pp. 140-165.

KLÍMOVÁ, E. Ordinamento lineare dell'enunciato in italiano. In Linguistica Pragensia, XII/1: 40-49, 2002.

L'imperativo italiano tra morfologia e pragmatica. In Études romanes de Brno, L 25: 85-96, 2004.

. Fattori della prospettiva funzionale dell'enunciato in italiano a confronto dell'inglese e del ceco. In KLÍMOVÁ, E. (ed.). In onore di Ivan Seidl. Opava: Slezská univerzita, 2007, pp. 113-123.

. Principi dell'ordine delle parole in tre lingue tipologicamente diverse. In RÜCKL, M.;

SANTORO, E; VEDDER, I. (eds.). Contesti di apprendimento di italiano L2. Firenze: Franco Cesati ed., 2013,pp. 43-57.

LEECH, G. N. Principles of Pragmatics, London, Longman, 1983.

STATI, S. Il dialogo. Considerazioni di linguistica pragmatica, Napoli, Liguori, 1982.

WITTGENSTEIN, L. Filosofická zkoumání (Ricerche filosofiche), Praha: AV ČR, 1993. 